\title{
Causal Inference with Genetic Data: Past, Present, and Future
}

\author{
Jean-Baptiste Pingault, ${ }^{1}$ Rebecca Richmond, ${ }^{2}$ and George Davey Smith ${ }^{2}$ \\ ${ }^{1}$ Division of Psychology and Language Sciences, University College London, London WC1H OAP \\ United Kingdom \\ ${ }^{2}$ MRC Integrative Epidemiology Unit, Population Health Sciences, Bristol Medical School, University of Bristol, \\ Bristol BS8 1TH, United Kingdom \\ Correspondence: j.pingault@ucl.ac.uk
}

\begin{abstract}
The set of methods discussed in this collection has emerged from the convergence of two scientific fields-genetics and causal inference. In this introduction, we discuss relevant aspects of each field and show how their convergence arises from the natural experiments that genetics offer. We present introductory concepts useful to readers unfamiliar with genetically informed methods for causal inference. We conclude that existing applications and foreseeable developments should ensure that we rapidly reap the rewards of this relatively new field, not only in terms of our understanding of human disease and development, but also in terms of tangible translational applications.
\end{abstract}

\begin{abstract}
dentifying causal risk and protective factors for human disease and development is a critical endeavor across social and biomedical sciences. Examples of causal questions that can be interrogated using the methods discussed in this collection include the following: Does vitamin D protect against multiple sclerosis (Mokry et al. 2015)? Does Selenium supplementation protect against cancer (Kho et al. 2019)? Do elevated inflammation biomarkers such as C-reactive protein increase the risk of depression (Prins et al. 2016)? Do high levels of circulating testosterone increase bone mineral density and decrease body fat (Mohammadi-Shemirani et al. 2020)? Does maternal smoking during pregnancy lower birth weight or increase the risk of child attention-deficit hyperactivity disorder (Thapar
\end{abstract}

et al. 2009)? Does higher education worsen myopia (Mountjoy et al. 2018)? Does victimization worsen adolescent mental health (Singham et al. 2017)? Does a tuberculosis infection increase the risk of lung adenocarcinoma (Wong et al. 2020)? Or, as an example of particular interest at the time of writing, can inflammatory biomarkers such as IL- 6 be targeted to decrease the risk of severe outcomes following SARS-CoV-2 infection (Bovijn et al. 2020)?

These questions only begin to capture the wide array of modifiable risk factors that can be investigated, including dietary supplements, biomarkers, lifestyles, social environments, or infections. More elaborate causal questions can also be asked by jointly modeling several risk factors. For example, is high-density lipoprotein

Editors: George Davey Smith, Rebecca Richmond, and Jean-Baptiste Pingault

Additional Perspectives on Combining Human Genetics and Causal Inference to Understand Human Disease and Development available at www.perspectivesinmedicine.org

Copyright (C) 2021 Cold Spring Harbor Laboratory Press; all rights reserved

Advanced Online Article. Cite this article as Cold Spring Harb Perspect Med doi: 10.1101/cshperspect.a041271 
J.-B. Pingault et al.

cholesterol (HDL-C) really "good cholesterol" (i.e., does it independently protect against coronary heart disease [CHD], even after accounting for the effect of other lipoproteins) (Davey Smith and Phillips 2020)? Or, what is the role of epigenetic markers (such as DNA methylation) in mediating the effect of obesity on cardiometabolic diseases (Mendelson et al. 2017)?

Answering those questions not only provides insights into the etiology of human disease and development but can also directly inform interventions. Conversely, inaccurate answers can lead to costly research dead ends and adverse public health consequences, such as the widespread consumption of inefficient and potentially iatrogenic supplements. Causal inference methods can be implemented to help answer those questions, by first providing evidence for or against the existence of a causal effect and by identifying its direction and estimating its magnitude.

In the articles in the Cold Spring Harbor Perspectives in Medicine subject collection "Combining Human Genetics and Causal Inference to Understand Human Disease and Development," we focus on a subset of such methods that use genetic data to strengthen causal inference in observational studies. We note that philosophers and scientists have grappled with the notion and definition of cause over centuries. We do not aim to contribute to this debate. Instead, in this collection, we focus on causal inference methods as tools to identify modifiable factors that, when changed, should lead to a change in the outcome of interest.

This collection has emerged from the convergence of two scientific fields-genetics and causal inference. We will discuss each in turn before demonstrating how they have converged to feed into genetically informed causal inference methods.

\section{FROM MENDELIAN TO MOLECULAR GENES}

Modern genetics can be traced back to Gregor Mendel's experimental work, published in 1866 , and the suggestion that discrete entities randomly transmitted across generations can explain the inheritance of discrete phenotypic (i.e., nongenetic) features, such as the color of peas (Mendel 1993). The focus on complex continuous traits such as human height came with the biometricians Francis Galton (Galton 1889) and Karl Pearson (Pearson and Henrici 1896), from the latter decades of the 19th to the start of the 20th century. Rather than from breeding experiments, within the context of human traits, biometricians used observed trait associations between family members to derive the role of genetic influences. Genetic influences were quantified using the concept of correlation introduced by Galton and formalized by Pearson.

The apparent paradox of discrete entitiesthe genes-having to account not only for discrete characteristics but also continuous traits initially divided Mendel's disciples and biometricians. Several contributions early in the 20th century contributed to the resolution of this debate (see Yule 1902; Visscher 2013). In 1911, Brownlee stated explicitly that "there is nothing necessarily antagonistic between the evidence advanced by the biometricians and the Mendelian theory" (Brownlee 1911). He showed that discrete elements consistent with the Mendelian theory could result in a normal distribution, concluding: "If the inheritance of stature depends upon a Mendelian mechanism, then the distribution of the population as regards height will be that which is actually found, namely, a distribution closely represented by the normal curve" (Brownlee 2013). In 1918, Ronald Fisher proposed an extended model including environmental effects in addition to many discrete genetic variants, or "cumulative Mendelian factors" (Fisher 1918). Fisher's extended model showed how the resulting phenotypic variance of complex traits could be partitioned into genetic and nongenetic components, laying the foundations of the field of quantitative genetics. Quantitative genetics has developed considerably since then by relying on the known genetic relatedness between relatives (e.g., twins) to better understand the respective importance of genetic and environmental factors and their interplay in explaining individual differences. Such etiological studies partition the variance of a single trait or the covariance between traits into genetic, shared, and nonshared environmental compo- 
nents (the nonshared component conflates external environmental influences but also measurement error and a likely substantial component due to variation explained by the intrinsic stochasticity of molecular processes) (Davey Smith 2011a; Plomin et al. 2013; Tikhodeyev and Shcherbakova 2019; Jonsson et al. 2021).

Following the discovery of DNA as the substrate for genes, followed by the uncovering of the structure of DNA, the notion of "genes" has evolved from "Mendelian genes" - abstract statistical entities explaining inheritance-to incarnate sequences of DNA or "molecular genes." These discoveries sparked the quest to identify molecular genes associated with diseases and traits. Among methods used to this end, genome-wide association studies (GWAS) have played a key role in the past 15 years. GWAS test the association of millions of genetic variants, typically single-nucleotide polymorphisms (SNPs), with a given trait. A vast array of downstream analyses can then be implemented to identify the genetic variants causing the disease. Analyses to identify causal variants in humans have typically relied on statistical methods, for example, testing which genetic variant stays the most associated to the trait after accounting for neighboring genetic variants (so-called "conditional analysis”) (Yang et al. 2012). The advent of genome editing methods so precise that they can edit out and/or replace a few genetic variants in vivo (Jinek et al. 2012) offers exciting opportunities to identify causal variants experimentally. The downstream effects of changing a given variant can be directly tested, consistent with the aforementioned notion that a risk factor is causal for an outcome when changing the risk factor also changes the outcome. Recent methods in the area hold considerable promise in uncovering the true causal variants and genes leading to diseases (Broekema et al. 2020).

Although the focus of the molecular era has mainly been to identify causal genetic variants, it has profoundly changed quantitative genetics and our ability to study the genetic architecture of traits. Genetic relatedness between distantly related individuals can also be calculated based on genome-wide markers. Similarly to familybased studies, this can then be exploited to derive the role of genetics in the variance and covariance of traits. For example, SNP heritability is the proportion of variance in a trait explained by the additive effects of all measured SNPs (Yang et al. 2011). Based on those measured SNPs, additional methods can be implemented to estimate the genetic correlation (e.g., how correlated are the genetic factors underlying schizophrenia and bipolar disorder?) (Bulik-Sullivan et al. 2015). In turn, models based on genetic correlation matrices for many traits can help us to better understand the genetic architecture of families of traits (e.g., how psychiatric traits cluster into subsets that are closely genetically related) (Grotzinger et al. 2019; Peyre et al. 2021). Such advances that jointly model all measured SNPs largely mimic what was possible with familybased studies (i.e., estimating population-level statistics like heritabilities or genetic correlations). However, a decisive advantage of the molecular era is that the information available at the individual level is considerably richer. Instead of knowing the place of an individual in a particular pedigree (e.g., as a member of a twin pair), we have access to millions of genetic variants for that individual. The cumulative effect of genetic variants can be thus captured by a polygenic score for any given trait (i.e., an individual-level score computed by summing risk variants weighted by effect sizes derived from GWAS) (International Schizophrenia Consortium et al. 2009; Dudbridge 2013). Polygenic scores can be computed based on genome-wide data or a subset of variants (e.g., genome-wide significant variants). Current polygenic scores based on GWAS for height and education predict $24 \%$ and $11 \%$ of the variance in their respective phenotype (Lee et al. 2018; Yengo et al. 2018). Such scores can then be used in (multivariate) models to examine genetic influences on an array of traits (Krapohl et al. 2016, 2017). As individual-level variables, they can improve predictive models of disease (e.g., cardiovascular disease [Sun et al. 2021]) and may lead to clinical applications (Torkamani et al. 2018; Wray et al. 2021).

Of note is that, despite often being labeled "etiological," investigations decomposing the variance of traits into genetic and environmental components have little to do with the iden- 
J.-B. Pingault et al.

tification of causal risk factors. This is because, in any particular study, the variance explained by genetics may largely depend on the distribution of environmental factors; conversely, the variance explained by environmental factors may depend on allele frequency in the study population. The decomposition of variance is therefore local to a study population and can be entirely different from the true role of genetics and the environment in explaining trait variation for a given trait. Only with an assumption of strict additivity (i.e., genetic effects are the same across all environments and vice versa) can study estimates reflect the respective etiological role of genetic and environmental influences. As Lewontin put it, "In view of the terrible mischief that has been done by confusing spatiotemporally local analysis of variance with the global analysis of cause, I suggest that we stop the endless search for better methods of estimating useless quantities" (Lewontin 1974). As noted later, however, "the local objection" is not really an objection regarding causal interpretation, but rather regarding "the generalizability of particular research findings," which is not only a problem for the analysis of variance but for any supposedly causal analysis (Vreeke 2006). More fundamental, however, is the realization that high heritability estimates may simply reflect a restricted range of observed environmental conditions in a given study. Changing environmental factors, for example, by intervention, can therefore still shift the distribution of a trait despite low estimates of environmental influences. Thus, heritability estimates say little about the malleability of traits to change; they reflect what is, rather than what could be. In addition, the concepts of heritable and environmental factors remain abstract in the sense that they do not identify specific modifiable factors that can be targeted for intervention, as is essential in useful causal analysis. This is true not only of classic methods of analysis of variance (e.g., twin heritability) referred to by Lewontin but also of newer methods such as SNP heritability, which estimate the variance explained by all common SNPs, rather than identify specific genetic variants.

Despite their importance, this collection does not focus on methods aiming to elucidate the genetic architecture of traits or identify causal genetic variants. Instead, we focus on the use of genetics as a powerful tool for establishing causal relationships at the phenotypic level. We aim to delineate how specific phenotypic risk factors cause phenotypic outcomes. That said, methods aiming to elucidate the genetic architecture of traits or identify causal genetic variants provide an essential background to the methods presented in this collection.

\section{CAUSAL INFERENCE IN OBSERVATIONAL DATA}

Randomized experiments and their implementation in clinical medicine as randomized controlled trials (RCTs) have come to be considered the gold standard for causal inference. The fundamental intuition is that if a treatment is allocated randomly to different units (e.g., human participants), then the treatment and control group will only differ due to the treatment. Comparing treatment and control groups on any outcome of interest (e.g., disease) thus allows us to establish the causal effect of the treatment and estimate its magnitude. Establishing causation has become so intertwined with experimentation and randomization that mentioning the "C-word" within observational research has been largely taboo in some fields (Hernán 2018; Grosz et al. 2020). That said, as discussed below, this taboo has been far from absolute with key contributions to causal reflection and modeling in observational settings in the second half of the 20th century. Still, authors are regularly compelled by journal policies to change wording from "effect" or "impact" to "association" or "link," thwarting the need for explicit and transparent reporting of the aim and methods of causal inference studies (Hernán 2018). Such a rigid application of the mantra "correlation is not causation" has long contributed to stalling the debate on causal inference in observational settings. Reducing causal inference to experimentation is not tenable. First, RCTs have their own limitations, for example, they may be not be ethical or feasible and it may be difficult to generalize their findings (Imai et al. 2008; Deaton and Cartwright 2018). Yet, public health may require that a pragmatic, 
even if imperfect, consensus is reached on the causal status of a given risk factor. In such cases, simply computing observed correlations between variables is often unhelpful and investigations within an explicit causal inference framework are required.

For example, no one now contests that smoking cigarettes is a causal risk factor for lung cancer. The causal status of smoking was hotly debated and contested by some including R.A. Fisher on the basis that confounding (Box 1)-including genetic confounding-prevented causal inference in observational data (Fisher 1957, 1958). But such objections were discarded based on converging observational evidence. In particular, Jerome Cornfield argued that the strength of confounding from genetic factors or other confounders would need to be implausibly high to account for all of the observed effect of smoking and cancer (Cornfield et al. 1959), laying the foundations of what is now known as sensitivity analysis. Concluding the debate, the report of the Royal College of Physicians in 1962 and the Surgeon General's Report in 1964 reached a consensus on the causal status of smoking, paving the way for largescale prevention efforts (Evans 1962; Terry 1964). This conclusion was attained without randomly allocating human participants to smoking, which would have been unethical.

Since then, and despite the aforementioned resistance, causal inference in observational settings has been continuously refined and formalized with inputs from both statistics and epidemiology. In 1965, Bill Cochran reflected on how to plan observational studies when experimentation is not possible "to elucidate causeand-effect relationships, or at least to investigate the relationships between one set of specified variables $X_{i}$ and a second set $Y_{i}$ in a way that suggests or appraises hypotheses about causation" (Cochran 1965). The choice of words is enlightening here: inference in observational settings may not provide definitive answers but can shift the cursor on a continuum from correlation to virtual causal certainty. Also, in 1965, Bradford Hill set out a list of viewpoints to consider when appraising empirical evidence in favor of or against a causal hypothesis, including temporal relationships, dose-response relationships, and plausibility (Hill 1965). Importantly, Hill's list was later misconstrued as a set of "criteria" to establish causality, a mechanical terminology he neither endorsed nor-it is clear-advocated (Davey Smith 2019).

In his words: "Here, then, are nine different viewpoints from all of which we should study association before we cry causation. What I do not believe-and this has been suggested-is that we can usefully lay down some hard-andfast rules of evidence that must be obeyed before we accept cause and effect. None of my nine viewpoints can bring indisputable evidence for or against the cause-and-effect hypothesis and none can be required as a sine qua non. What they can do, with greater or less strength, is to help us to make up our minds on the fundamental question - is there any other way of explaining the set of facts before us, is there any other answer equally, or more likely than cause and effect?" In the same text, Hill also criticized the overreliance on tests of significance, suggesting that in some cases, descriptive tables are so clear that such tests do not add any value, or that "the glitter" of "magic formulae" can divert our attention from substantial study shortcomings. He concluded, "Like fire, the $\chi^{2}$ test is an excellent servant and a bad master."

Hill's informal approach to causal inference was later criticized (see Rothman 2020) as none of his viewpoints is sufficient or necessary to infer causality, which he himself recognized (on the tension between Hill's criteria and statistical formalization, see Rothman 2020 and VanderWeele 2020). The most influential formal causal inference frameworks to date are arguably Donald Rubin's potential outcomes framework and Judea Pearl's structural models (Pearl 2009; Imbens and Rubin 2015). Both frameworks are very general, and most causal inference designs or statistical methods in observational settings (and even randomized trials) can be subsumed under these frameworks. Both formalize assumptions under which causal estimates can be attainable from observational data. Exchangeability is a fundamental notion in both frameworks and is achieved when exposed and nonexposed groups are balanced on all confounders, 
J.-B. Pingault et al.

\section{BOX 1. CAUSAL DIAGRAMS}

Directed acyclic graphs (DAGs) can be used to encode causal models and assumptions. (i) The directed arrow represents a causal effect of A on B. (ii) The directed path goes from A to C via B. B is here a mediator, in the sense that the causal effect of $A$ on $C$ is happening indirectly via B. In DAGs, absent arrows are as important as represented arrows. In $i i$, for example, we assume that all the effect of $A$ on $C$ is mediated by $B$ (i.e., there is no additional arrow directly from $A$ to $C$ or via another variable than B). (iii) C independently causes A and B. C is therefore a confounder of the association between $A$ and B. The path between A and B via C is called a "backdoor" path. Such a backdoor path creates an observed association between $A$ and $B$ even in the absence of a causal effect, which is represented by the absence of a directed arrow between $A$ and $B$. This constitutes a fundamental challenge in epidemiology as observed associations between two variables cannot be assumed to stem from a causal relationship between those two variables. If $\mathrm{C}$ has been observed and perfectly measured, then statistically adjusting for $C$ will remove confounding and enable the estimation of the causal effect between $A$ and $B$, here a null effect.

Directions of arrows matter in DAGs. For example, if the arrow between $\mathrm{A}$ and $\mathrm{C}$ is reversed in iii then $\mathrm{C}$ becomes a mediator rather than a confounder. (iv) $\mathrm{C}$ is a collider as both arrows from $\mathrm{A}$ and $\mathrm{B}$ "collide" on C. In this situation, the path is blocked by C. As such, and contrary to the confounder situation, there is no observed association between $\mathrm{A}$ and $\mathrm{B}$. However, if $\mathrm{C}$ is adjusted for, this may create a spurious association between $\mathrm{A}$ and $\mathrm{B}$. This collider bias is another key challenge in epidemiology. If $\mathrm{C}$ is a collider but mistakenly identified as a confounder, the adjusted association may be further from the causal effect than the unadjusted association. Collider bias can also generate bias in many study settings. For example, if two independent factors ( $A$ and $B$ ) cause hospitalization $(C)$, then, in a study restricted to hospitalized patients, $A$ and $B$ will generally be associated. This is because the stratification (i.e., focusing only on hospitalized rather than hospitalized and nonhospitalized people) is a form of adjustment.

( $v$ ) $\mathrm{C}$ is a confounder of $\mathrm{X}$ and $\mathrm{Y}$ and should therefore be adjusted for to retrieve the causal effect of $\mathrm{X}$ on $\mathrm{Y}$. However, $\mathrm{C}$ is also a collider of $\mathrm{A}$ and $\mathrm{B}$. Adjusting for $\mathrm{C}$ thus creates a spurious association between $A$ and $B$, which introduces a backdoor path from $X$ to $Y$ via $A$ and $B$. The induced association upon confounder adjustment in this context has been referred to as "M bias." In addition to adjusting for $\mathrm{C}$, it is thus necessary to adjust for $\mathrm{A}$ and/or B to further block the newly created path. Importantly, in theory, if the model DAG corresponds to the true model, finding a sufficient set of confounders, here $\mathrm{C}$ and $\mathrm{A}$ (or $\mathrm{B}$ ) for example, is sufficient to retrieve the causal effect of $\mathrm{X}$ and $\mathrm{Y}$. In practice, however, we do not know the underlying causal model and the variables are not measured without error. This is a major impediment for causal inference based only on statistical adjustment, given the nature of epidemiological data where unclear underlying models, unmeasured confounders, and measurement error are the norm. In this collection, we present a number of methods that (partly) adjust by design for unobserved confounders (e.g., the twin design).

The DAG (vi) encodes the instrumental variable design. $Z$ is the instrument, which is used in an "instrumental" fashion to estimate the causal effect of $X$ on $Y$. Note that the DAG encodes three assumptions of the instrumental variable approach that are necessary for $Z$ to be a valid instrument, enabling the inference that $X$ causes $Y$. First, $Z$ needs to be (robustly) associated with $X$ (blue arrow), which is called the relevance assumption (i.e., $Z$ needs to be relevant to assess the effect of $X$ ). The second assumption is exchangeability and is encoded by the absence of a common cause of $Z$ and $Y$. Exchangeability is key to understand why $X$ enables us to make causal inference regarding $X$ to $Y$. To illustrate, if $Z$ is binary and positively predicts $X$, then participants in group $Z_{1}$ will have higher levels of $X$ than participants $Z_{0}$. Although they differ on the level of $X$, participants $Z_{0}$ and $Z_{1}$ do not differ on any other variables. Participants $Z_{0}$ and $Z_{1}$ are thus exchangeable and only differ on the exposure $X$. If $Z_{0}$ and $Z_{1}$ have different outcomes (i.e., different levels of $Y$ ), we can conclude that $X$ is causally related to $Y$. This is similar to a randomized controlled trial (RCT) in the sense that $Z$ plays the role of the random assignment, which creates two groups with a different level of the variable influenced by the treatment $X$ but balanced on all other confounders. Third, Z needs to be associated with Y only via its effect on $X$, which is called the exclusion restriction assumption. In other words, 
similarly to DAG ii, X fully mediates the association between $\mathrm{Z}$ and $\mathrm{Y}$. When using a genetic instrument, this is often called mediated pleiotropy (or vertical pleiotropy) as opposed to unmediated (or horizontal) pleiotropy, which would be represented by a direct arrow from Z to Y. Importantly, even when the DAG in vi fully holds, we do expect an observed association between $Z$ and $Y$, which is equal to the path from $Z$ to $Y$ via $X$.

(i) $\mathrm{A} \longrightarrow \mathrm{B}$

(ii) $\mathrm{A} \longrightarrow \mathrm{B} \longrightarrow \mathrm{C}$

(iii) $\mathrm{A} \longleftrightarrow \mathrm{C} \longrightarrow \mathrm{B}$

(iv)

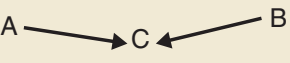

(v)
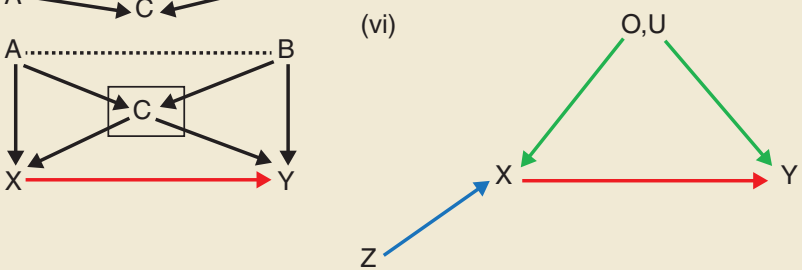

as occurs in an appropriately randomized trial. Causal models within those frameworks can be conveniently represented in formal diagrams, or "directed acyclic graphs" (DAGs) (Box 1). Both frameworks have a dedicated statistical notation that is considerably more sophisticated than statistics like the $t$-test and chi-squared test referred to by Hill.

The divide between empiricists like Hill and advocates of the primacy of formal statistical frameworks is unwarranted. First, the focus of both sides is somewhat different. Hill's address was aimed at practitioners of occupational medicine, with a strong focus on pragmatic decision making. He concludes his address by a case for action: although scientific knowledge is by nature incomplete, acting on such knowledge should not be endlessly deferred. Conversely, formalists focus on methodological advances aiming to provide the best answer to a causal question and estimates of causal effects under a given set of assumptions. However, even the most sophisticated causal models in observational data can only yield the right causal estimates when the specified model is mostly correct (e.g., a sufficient set of confounders have been assessed accurately; see Box 1). Substantive prior knowledge is required to specify appropriate models and assess their assumptions, the plausibility of their findings, and even to formulate relevant causal questions in the first place. That is, causal inference cannot be reduced to algorithms (Krieger and Davey Smith 2018). In turn, however, formalized tools remove some unwarranted arbitrariness in the decisionmaking process regarding the causal status of risk factors (Baiocchi 2020; VanderWeele 2020). As in all empirical sciences, a constant dialogue must be maintained between theoretical frameworks, statistical methods, and empirical evidence.

\section{GENETICS AND PHENOTYPIC CAUSAL INFERENCE}

Genetics and causal inference have developed largely in parallel but have converged along two lines of inquiry. First, family-based designs used in quantitative genetics to understand the genetic and environmental architecture of traits have also been used explicitly for causal inference. The idea of using twins that are genetically identical to identify environmental causes of diseases has been present since the 1950s, with, for example, an analysis of smoking habits in twins that concluded that a sufficient number of discordant twins would help in establishing the injurious effect of tobacco smoking (Friberg et al. 1959), which was confirmed much later (Kaprio and Koskenvuo 1989; Hjelmborg et al. 2017). The approach has then been systematized on large twin samples, based on the principle 
J.-B. Pingault et al.

that identical twins exposed to a risk factor can be matched with their nonexposed cotwins (Carlin et al. 2005; McGue et al. 2010), enhancing exchangeability. Other family-based designs such as the in vitro fertilization design can be used to account for genetic confounding by comparing genetically related and genetically unrelated parent-child pairs (Thapar et al. 2009). Many such methods and examples of applications are proposed in this collection.

Second, and more recently, measured genetic variants associated with an exposure (e.g., cholesterol) have been used as instruments (Box 1) to estimate the causal effect of that exposure on relevant outcomes (e.g., cardiovascular diseases). This approach was named Mendelian randomization (MR), as it capitalizes on the randomization of genetic material occurring at conception to approximate exchangeability and strengthen inference (Davey Smith and Ebrahim 2003). Interestingly, the idea that randomization at conception can help for controlled comparison had been grasped by Fisher. Indeed, Fisher himself highlighted the connectedness between his central contribution to the statistics of randomized experiments and Fisher's early work on the transmission of Mendelian factors (Davey Smith 2011b). In his words,

And here I may mention a connection between our two subjects which seem not to be altogether accidental, namely that the factorial method of experimentation, now of lively concern so far afield as the psychologists, or the industrial chemists, derives its structure and its name, from the simultaneous inheritance of Mendelian factors... Genetics is indeed in a peculiarly favoured condition in that Providence has shielded the geneticist from many of the difficulties of a reliably controlled comparison. The different genotypes possible from the same mating have been beautifully randomised by the meiotic process. A more perfect control of conditions is scarcely possible, than that of different genotypes appearing in the same litter. (Fisher 1952)

As such, MR can be construed as a return to the roots of causal inference; it has developed considerably over the past decade with a flurry of methods and applications reviewed in this collection.

\section{CONTRIBUTIONS}

Lynch's (2021) contribution discusses the specific meaning of "cause" in genetics from a philosophical perspective, building on the distinction between Mendelian and molecular genes. The rest of this collection focuses more pragmatically on describing genetically informed methods for causal inference and their applications. Thapar and Rice (2021) present a range of family-based designs for causal inference while McAdams et al. (2021) focus on the twin design and its extensions to larger pedigrees. Richmond and Davey Smith (2021) turn to explaining the fundamentals of MR and how genetic variants and in particular SNPs can be used as instruments for causal inference. Dudbridge (2021) follows up on the many extensions of MR jointly modeling many SNPs as instruments. Sanderson (2021) focuses on a special case of polygenic MR using instruments associated with several exposures to identify the independent or mediating effects of such exposures on outcomes of interest. Although the two major lines of research using genetics for causal inference (i.e., family-based and MR) have emerged and evolved independently, Hwang et al. (2021) outline the major opportunities arising from their recent integration. To some extent, family-based MR returns to Fisher's insight of randomization of genotypes in the same litter.

Note that we define phenotypes broadly as including any individual characteristic other than genotypes, which includes all omics other than genomics. Methods covered in Porcu et al. (2021) aim to query the potential of genetically informed methods in elucidating the role of metabolomics as modifiable risk factors for diseases. Such research questions build on largescale and growing data sets and Richardson et al. (2021) cover much-needed computational tools for causal inference.

Experimental and observational causal inference methods have often been artificially opposed. However, formal causal inference languages subsume both under the same theoretical frameworks and notations and, in practice, they can and should be complementary. Ference et al. (2021) show how MR can be used to improve the 
design of randomized control trials and Schmidt et al. (2021) how genetics can be used to prioritize drug targets for trials.

Naturally, each of the methods covered in this collection has its own challenges and limitations. Munafò et al. (2021) conclude this collection by reflecting on how triangulation of evidence from multiple genetically informed and nongenetic methods can help in further strengthening causal inference.

\section{TRENDS AND FUTURE DEVELOPMENTS}

Following a more succinct attempt (Pingault et al. 2018), this collection is the first to comprehensively cover genetically informed designs for causal inference. New trends are already apparent and should further develop in the near future. We expect that the development of new methods or the refinement of existing ones will continue at a fast pace. At the theoretical level, classical models such as the discordant twin models should be rewritten using more formal causal inference language to better understand their underlying assumption and the meaning of the resulting causal estimates (Petersen and Lange 2020). Ever more robust MR estimators are continuously being developed. In particular, new methods leveraging genome-wide data for causal inference are emerging and should become a powerful viable complement to current approaches that use a few dozen or hundreds of genetic variants as instruments (Darrous et al. 2020; Morrison et al. 2020). Emerging methods further discussed by Hwang et al. (2021) in this collection, like MR within families, offer to address some of the shortcomings of MR (Howe et al. 2021). In addition, these approaches offer new opportunities to further examine old questions such as what underlying processes explain the transmission of risk across generations. Intergenerational causal inference can elucidate whether parental risk factors have causal effects on offspring outcomes or whether intergenerational associations are better explained by genetic and environmental confounding (Lawlor et al. 2017; Kong et al. 2018; Balbona et al. 2020).

So far, quantitative genetic methods have largely relied on controlling for confounding to strengthen inference, whereas methods using molecular genetic data like MR have relied on instrumental variable approaches (Box 1). New methods can arise from crossing these boundaries. For example, genetic scores can be used as instruments within the twin design (Minică et al. 2018). Genetically informed methods can also be combined with more classical methods for causal inference. For example, MR can be combined with negative control analyses (Sanderson et al. 2021). Genome-wide polygenic scores can be used to implement genetically informed sensitivity analyses (Pingault et al. 2018, 2021), building on the concept of sensitivity analysis that emerged from the work of Jerome Cornfield during the smoking-lung cancer controversy.

The scope of application of methods presented in this collection becomes wider as new data sets are made available. The emerging literature on the genetic architecture of COVID19 , made possible by the data collected by the COVID-19 Host Genetics Initiative offers a good example of how methods presented in this collection can make decisive contributions to emerging questions. A genetic instrument for IL-6R was found to be associated with a lower risk of hospitalization for COVID-19, suggesting the relevance of therapeutic inhibition of IL6R, which has now been confirmed in clinical trials (Bovijn et al. 2020; The WHO Rapid Evidence Appraisal for COVID-19 Therapies (REACT) Working Group et al. 2021). MR has also been used to systematically scan hundreds of druggable proteins to prioritize targets for drug trials, for example, highlighting OAS1 as a candidate for drug development (Gaziano et al. 2021; Zhou et al. 2021). Additional studies point toward host antiviral defense mechanisms and mediators of inflammatory organ damage as mechanisms underlying critical illness in COVID-19 (Pairo-Castineira et al. 2021).

Although genetically informed causal inference is relatively recent, we expect that we will start reaping rewards in the near future, that is, not only in terms of our understanding of human disease and development but in terms of tangible translational applications such as drug development. 
J.-B. Pingault et al.

\section{REFERENCES}

${ }^{*}$ Reference is also in this collection.

Baiocchi M. 2020. Following Bradford Hill, in reprint of Hill's "the environment and disease: association or causation?" and comments. Obs Stud 6: 1-9.

Balbona J, Kim Y, Keller MC. 2020. Estimation of parental effects using polygenic scores. Behav Genet 51: 264-278. doi:10.1007/s10519-020-10032-w

Bovijn J, Lindgren CM, Holmes MV. 2020. Genetic variants mimicking therapeutic inhibition of IL-6 receptor signaling and risk of COVID-19. Lancet Rheumatol 2: e658e659. doi:10.1016/S2665-9913(20)30345-3

Broekema RV, Bakker OB, Jonkers IH. 2020. A practical view of fine-mapping and gene prioritization in the post-genome-wide association era. Open Biol 10: 190221. doi:10 $.1098 /$ rsob.190221

Brownlee J. 1911. The inheritance of complex growth forms, such as stature, on Mendel's theory. Proceedings of the Royal Society of Edinburgh (reprinted in 2013, Int J Epidemiol 42: 932-934).

Bulik-Sullivan BK, Loh PR, Finucane HK, Ripke S, Yang J; Schizophrenia Working Group of the Psychiatric Genomics Consortium; Patterson N, Daly MJ, Price AL, Neale BM. 2015. LD score regression distinguishes confounding from polygenicity in genome-wide association studies. Nat Genet 47: 291-295. doi:10.1038/ng.3211

Carlin JB, Gurrin LC, Sterne JA, Morley R, Dwyer T. 2005. Regression models for twin studies: a critical review. Int $J$ Epidemiol 34: 1089-1099. doi:10.1093/ije/dyi153

Cochran WG. 1965. The planning of observational studies of human populations. J R Stat Soc Ser Gen 128: 234-266. doi: $10.2307 / 2344179$

Cornfield J, Haenszel W, Hammond EC, Lilienfeld AM, Shimkin MB, Wynder EL. 1959. Smoking and lung cancer: recent evidence and a discussion of some questions. $J$ Natl Cancer Inst 22: 173-203.

Darrous L, Mounier N, Kutalik Z. 2020. Simultaneous estimation of bi-directional causal effects and heritable confounding from GWAS summary statistics. medRxiv doi:10.1101/2020.01.27.20018929

Davey Smith G. 2011a. Epidemiology, epigenetics and the "gloomy prospect": embracing randomness in population health research and practice. Int J Epidemiol 40: 537-562. doi:10.1093/ije/dyr117

Davey Smith G. 2011b. Commentary: random allocation in observational data: how small but robust effects could facilitate hypothesis-free causal inference. Epidemiology 22: 460-463. doi:10.1097/EDE.0b013e31821d0426

Davey Smith G. 2019. Post-modern epidemiology: when methods meet matter. Am J Epidemiol 188: 1410-1419. doi:10.1093/aje/kwz064

Davey Smith G, Ebrahim S. 2003. "Mendelian randomization": can genetic epidemiology contribute to understanding environmental determinants of disease? Int $J$ Epidemiol 32: 1-22.

Davey Smith G, Phillips AN. 2020. Correlation without a cause: an epidemiological odyssey. Int J Epidemiol 49: 4-14. doi:10.1093/ije/dyaa016
Deaton A, Cartwright N. 2018. Understanding and misunderstanding randomized controlled trials. Soc Sci Med 210: 2-21. doi:10.1016/j.socscimed.2017.12.005

Dudbridge F. 2013. Power and predictive accuracy of polygenic risk scores. PLoS Genet 9: e1003348. doi:10.1371/ journal.pgen.1003348

* Dudbridge F. 2021. Polygenic Mendelian randomization. Cold Spring Harb Perspect Med 11: a039586. doi:10 .1101/cshperspect.a039586

Evans PA. 1962. Smoking and health. Summary of a report of the Royal College of Physicians of London on smoking in relation to cancer of the lung and other diseases. Cent Aft J Med 8: 234-236.

* Ference BA, Holmes MV, Davey Smith G. 2021. Using Mendelian randomization to improve the design of randomized trials. Cold Spring Harb Perspect Med 11: a040980. doi:10.1101/cshperspect.a040980

Fisher RA. 1918. The correlation between relatives on the supposition of Mendelian inheritance. Trans R Soc Edinb 52: 399-433. doi:10.1017/S0080456800012163

Fisher R. 1952. Statistical methods in genetics. Heredity 6 1-12 (reprinted in 2010, Int J Epidemiol 39: 329-335).

Fisher RA. 1957. Alleged dangers of cigarette-smoking. BMJ 2: 297-298. doi:10.1136/bmj.2.5039.297-b

Fisher R. 1958. Cigarettes, cancer, and statistics. Centen Rev Arts Sci 2: 151-166.

Friberg L, Kaij L, Dencker SJ, Jonsson E. 1959. Smoking habits of monozygotic and dizygotic twins. $\mathrm{Br} \mathrm{Med} \mathrm{J} \mathrm{1:}$ 1090-1092. doi:10.1136/bmj.1.5129.1090

Galton F. 1889. Natural inheritance. Macmillan, New York.

Gaziano L, Giambartolomei C, Pereira AC, Gaulton A, Posner DC, Swanson SA, Ho YL, Iyengar SK, Kosik NM, Vujkovic M, et al. 2021. Actionable druggable genomewide Mendelian randomization identifies repurposing opportunities for COVID-19. Nat Med 27: 668-676. doi:10.1038/s41591-021-01310-z

Grosz MP, Rohrer JM, Thoemmes F. 2020. The taboo against explicit causal inference in nonexperimental psychology. Perspect Psychol Sci 15: 1243-1255. doi:10.1177/ 1745691620921521

Grotzinger AD, Rhemtulla M, de Vlaming R, Ritchie SJ, Mallard TT, Hill WD, Ip HF, Marioni RE, McIntosh AM, Deary IJ, et al. 2019. Genomic structural equation modelling provides insights into the multivariate genetic architecture of complex traits. Nat Hum Behav 3: 513 525. doi:10.1038/s41562-019-0566-x

Hernán MA. 2018. The C-word: scientific euphemisms do not improve causal inference from observational data. Am J Public Health 108: 616-619. doi:10.2105/AJPH.2018 .304337

Hill AB. 1965. The environment and disease: association or causation? Proc R Soc Med 58: 295-300.

Hjelmborg J, Korhonen T, Holst K, Skytthe A, Pukkala E, Kutschke J, Harris JR, Mucci LA, Christensen K, Czene K, et al. 2017. Lung cancer, genetic predisposition and smoking: the Nordic twin study of cancer. Thorax 72: 10211027. doi:10.1136/thoraxjnl-2015-207921

Howe LJ, Nivard MG, Morris TT, Hansen AG, Rasheed H, Cho Y, Chittoor G, Lind PA, Palviainen T, van der Zee $\mathrm{MD}$, et al. 2021. Within-sibship GWAS improve estimates 
of direct genetic effects. bioRxiv doi:10.1101/2021.03.05 .433935

* Hwang LD, Davies NM, Warrington NM, Evans DM. 2021. Integrating family-based and Mendelian randomization designs. Cold Spring Harb Perspect Med 11: a039503. doi:10.1101/cshperspect.a039503

Imai K, King G, Stuart EA. 2008. Misunderstandings between experimentalists and observationalists about causal inference. J R Stat Soc Ser A 171: 481-502. doi:10.1111/j .1467-985X.2007.00527.x

Imbens GW, Rubin DB. 2015. Causal inference for statistics, social, and biomedical sciences. Cambridge University Press, Cambridge.

International Schizophrenia Consortium; Purcell SM, Wray NR, Stone JL, Visscher PM, O’Donovan MC, Sullivan PF, Sklar P. 2009. Common polygenic variation contributes to risk of schizophrenia and bipolar disorder. Nature 460: 748-752. doi:10.1038/nature08185

Jinek M, Chylinski K, Fonfara I, Hauer M, Doundna JA, Charpentier E. 2012. A programmable dual-RNA-guided DNA endonuclease in adaptive bacterial immunity. Science 337: 816-821. doi:10.1126/science.1225829

Jonsson H, Magnusdottir E, Eggertsson HP, Stefansson OA, Arnadottir GA, Eiriksson O, Zink F, Helgason EA, Jonsdottir I, Gylfason A, et al. 2021. Differences between germline genomes of monozygotic twins. Nat Genet 53: 27-34. doi:10.1038/s41588-020-00755-1

Kaprio J, Koskenvuo M. 1989. Twins, smoking and mortality: a 12-year prospective study of smoking-discordant twin pairs. Soc Sci Med 29: 1083-1089. doi:10.1016/ 0277-9536(89)90020-8

Kho PF, Glubb DM, Thompson DJ, Spurdle AB, O'Mara TA. 2019. Assessing the role of selenium in endometrial cancer risk: a Mendelian randomization study. Front Oncol 9: 182. doi:10.3389/fonc.2019.00182

Kong A, Thorleifsson G, Frigge ML, Vilhjalmsson BJ, Young AI, Thorgeirsson TE, Benonisdottir S, Oddsson A, Halldorsson BV, Masson G, et al. 2018. The nature of nurture: effects of parental genotypes. Science 359: 424-428. doi:10.1126/science.aan6877

Krapohl E, Euesden J, Zabaneh D, Pingault JB, Rimfeld K, von Stumm S, Dale PS, Breen G, O’Reilly PF, Plomin R. 2016. Phenome-wide analysis of genome-wide polygenic scores. Mol Psychiatry 21: 1188-1193. doi:10.1038/mp .2015 .126

Krapohl E, Hannigan LJ, Pingault JB, Patel H, Kadeva N, Curtis C, Breen G, Newhouse SJ, Eley TC, et al. 2017. Widespread covariation of early environmental exposures and trait-associated polygenic variation. Proc Natl Acad Sci 114: 11727-11732. doi:10.1073/pnas.1707178114

Krieger N, Davey Smith G. 2018. Reply to Pearl: Algorithm of the truth vs real-world science. Int J Epidemiol 47: 1004-1006.

Lawlor D, Richmond R, Warrington N, McMahon G, Davey Smith G, Bowden J, Evans DM. 2017. Using Mendelian randomization to determine causal effects of maternal pregnancy (intrauterine) exposures on offspring outcomes: sources of bias and methods for assessing them. Wellcome Open Res 2: 11. doi:10.12688/wellcomeopenres .10567 .1

Lee JJ, Wedow R, Okbay A, Kong E, Maghzian O, Zacher M, Nguyen-Viet TA, Bowers P, Sidorenko J, Karlsson Linnér
R, et al. 2018. Gene discovery and polygenic prediction from a genome-wide association study of educational attainment in 1.1 million individuals. Nat Genet 50: 11121121. doi:10.1038/s41588-018-0147-3

Lewontin RC. 1974. The analysis of variance and the analysis of causes. Am J Hum Genet 26: 400-411 (reprinted in 2006, Int J Epidemiol 35: 520-525).

* Lynch KE. 2021. The meaning of "cause" in genetics. Cold Spring Harb Perspect Med doi:10.1101/cshperspect. a040519

* McAdams TA, Rijsdijk FV, Zavos HMS, Pingault JB. 2021. Twins and causal inference: leveraging nature's experiment. Cold Spring Harb Perspect Med 11: a039552. doi:10.1101/cshperspect.a039552

McGue M, Osler M, Christensen K. 2010. Causal inference and observational research: the utility of twins. Perspect Psychol Sci 5: 546-556. doi:10.1177/1745691610383511

Mendel G. 1993. Experiments on plant hybrids. In Gregor Mendel's experiments on plant hybrids: A guided study. Rutgers University Press, New Brunswick, NJ.

Mendelson MM, Marioni RE, Joehanes R, Liu C, Hedman AK, Aslibekyan S, Demerath EW, Guan W, Zhi D, Yao C, et al. 2017. Association of body mass index with DNA methylation and gene expression in blood cells and relations to cardiometabolic disease: a Mendelian randomization approach. PLoS Med 14: e1002215. doi:10.1371/ journal.pmed.1002215

Minică CC, Dolan CV, Boomsma DI, de Geus E, Neale MC. 2018. Extending causality tests with genetic instruments: an integration of Mendelian randomization with the classical twin design. Behav Genet 48: 337-349. doi:10.1007/ s10519-018-9904-4

Mohammadi-Shemirani P, Chong M, Pigeyre M, Morton RW, Gerstein HC, Paré G. 2020. Effects of lifelong testosterone exposure on health and disease using Mendelian randomization. eLife 9: e58914. doi:10.7554/eLife.58914

Mokry LE, Ross S, Ahmad OS, Forgetta V, Smith GD, Goltzman D, Leong A, Greenwood CM, Thanassoulis G, Richards JB. 2015. Vitamin D and risk of multiple sclerosis: a Mendelian randomization Study. PLoS Med 12: e1001866. doi:10.1371/journal.pmed.1001866

Morrison J, Knoblauch N, Marcus JH, Stephens M, He X. 2020. Mendelian randomization accounting for correlated and uncorrelated pleiotropic effects using genomewide summary statistics. Nat Genet 52: 740-747. doi:10 .1038/s41588-020-0631-4

Mountjoy E, Davies NM, Plotnikov D, Smith GD, Rodriguez S, Williams CE, Guggenheim JA, Atan D. 2018. Education and myopia: assessing the direction of causality by Mendelian randomisation. BMJ 361: k2022. doi:10.1136/bmj .$k 2022$

* Munafò MR, Higgins JPT, Davey Smith G. 2021. Triangulating evidence through the inclusion of genetically in formed designs. Cold Spring Harb Perspect Med 11: a040659. doi:10.1101/cshperspect.a040659

Pairo-Castineira E, Clohisey S, Klaric L, Bretherick AD, Rawlik K, Pasko D, Walker S, Parkinson N, Fourman MH, Russell CD, et al. 2021. Genetic mechanisms of critical illness in COVID-19. Nature 591: 92-98. doi:10.1038/ s41586-020-03065-y

Pearl J. 2009. Causality. Cambridge University Press, Cambridge. 
J.-B. Pingault et al.

Pearson K, Henrici OMFE. 1896. Mathematical contributions to the theory of evolution. III: Regression, heredity, and panmixia. Philos Trans R Soc Lond A 187: 253-318.

Petersen AH, Lange T. 2020. What is the causal interpretation of sibling comparison designs? Epidemiology 31: 7581. doi:10.1097/EDE.0000000000001108

Peyre H, Schoeler T, Liu C, Williams CM, Hoertel N, Havdahl A, Pingault JB. 2021. Combining multivariate genomic approaches to elucidate the comorbidity between autism spectrum disorder and attention deficit hyperactivity disorder. J Child Psychol Psychiatry doi:10.1111/jcpp .13479

Pingault JB, O'Reilly PF, Schoeler T, Ploubidis GB, Rijsdijk F, Dudbridge F. 2018. Using genetic data to strengthen causal inference in observational research. Nat Rev Genet 19: 566-580. doi:10.1038/s41576-018-0020-3

Pingault JB, Rijsdijk F, Schoeler T, Choi SW, Selzam S, Krapohl E, O'Reilly PF, Dudbridge F. 2021. Genetic sensitivity analysis: adjusting for genetic confounding in epidemiological associations. PLOS Genet 17: e1009590. doi:10 .1371/journal.pgen.1009590

Plomin R, DeFries JC, Knopik VS, Neiderhiser JM. 2013. Behavioral genetics. Worth, New York.

* Porcu E, Sjaarda J, Lepik K, Carmeli C, Darrous L, Sulc J, Mounier N, Kutalik Z. 2021. Causal inference methods to integrate omics and complex traits. Cold Spring Harb Perspect Med 11: a040493. doi:10.1101/cshperspect .a040493

Prins BP, Abbasi A, Wong A, Vaez A, Nolte I, Franceschini N, Stuart PE, Guterriez Achury J, Mistry V, Bradfield JP, et al. 2016. Investigating the causal relationship of C-reactive protein with 32 complex somatic and psychiatric outcomes: a large-scale cross-consortium Mendelian randomization study. PLoS Med 13: e1001976. doi:10.1371/ journal.pmed.1001976

* Richardson TG, Zheng J, Gaunt TR. 2021. Computational tools for causal inference in genetics. Cold Spring Harb Perspect Med 11: a039248. doi:10.1101/cshperspect .a039248

* Richmond RC, Davey Smith G. 2021. Mendelian randomization: concepts and scope. Cold Spring Harb Perspect Med doi:10.1101/cshperspect.a040501

Rothman KJ. 2020. The wrong message from the wrong talk. Obs Stud 6: 30-32.

* Sanderson E. 2021. Multivariable Mendelian randomization and mediation. Cold Spring Harb Perspect Med 11: a038984. doi:10.1101/cshperspect.a038984

Sanderson E, Richardson TG, Hemani G, Davey Smith G. 2021. The use of negative control outcomes in Mendelian randomization to detect potential population stratification. Int J Epidemiol doi:10.1093/ije/dyaa288

* Schmidt AF, Hingorani AD, Finan C. 2021. Human genomics and drug development. Cold Spring Harb Perspect Med doi:10.1101/cshperspect.a039230

Singham T, Viding E, Schoeler T, Arseneault L, Ronald A, Cecil CM, McCrory E, Fijsdijk F, Pingault JB. 2017. Concurrent and longitudinal contribution of exposure to bullying in childhood to mental health: the role of vulnerability and resilience. JAMA Psychiatry 74: 1112-1119. doi:10.1001/jamapsychiatry.2017 .2678
Sun L, Pennells L, Kaptoge S, Nelson CP, Ritchie SC, Abraham G, Arnold M, Bell S, Bolton T, Burgess S, et al. 2021. Polygenic risk scores in cardiovascular risk prediction: a cohort study and modelling analyses. PLoS Med 18: e1003498. doi:10.1371/journal.pmed.1003498

Terry LL. 1964. Smoking and health. Report of the advisory committee to the surgeon general of the public health service. Department of Health, Education, and Welfare, Washington, DC.

* Thapar A, Rice F. 2021. Family-based designs that disentangle inherited factors from pre- and postnatal environmental exposures: in vitro fertilization, discordant sibling pairs, maternal versus paternal comparisons, and adoption designs. Cold Spring Harb Perspect Med 11: a038877. doi:10.1101/cshperspect.a038877

Thapar A, Rice F, Hay D, Boivin J, Langley K, van den Bree M, Rutter M, Harold G. 2009. Prenatal smoking might not cause attention-deficit/hyperactivity disorder: evidence from a novel design. Biol Psychiatry 66: 722-727. doi:10.1016/j.biopsych.2009.05.032

The WHO Rapid Evidence Appraisal for COVID-19 Therapies (REACT) Working Group; Shankar-Hari M, Vale CL, Godolphin PJ, Fisher D, Higgins JPT, Spiga F, Savovic J, Tierney J, Baron G, et al. 2021. Association between administration of IL-6 antagonists and mortality among patients hospitalized for COVID-19: a meta-analysis. JAMA 326: 499-518. doi:10.1001/jama.2021.11330

Tikhodeyev ON, Shcherbakova OV. 2019. The problem of non-shared environment in behavioral genetics. Behav Genet 49: 259-269. doi:10.1007/s10519-019-09950-1

Torkamani A, Wineinger NE, Topol EJ. 2018. The personal and clinical utility of polygenic risk scores. Nat Rev Genet 19: 581-590. doi:10.1038/s41576-018-0018-x

VanderWeele TJ. 2020. Hill's causal considerations and the potential outcomes framework. Obs Stud 6: 47-54.

Visscher PM. 2013. Commentary: height and Mendel's theory: the long and the short of it. Int J Epidemiol 42: 944945. doi:10.1093/ije/dyt069

Vreeke GJ. 2006. Commentary: the attainability of causal knowledge of genetic effects in complex human traits Int J Epidemiol 35: 531-534. doi:10.1093/ije/dyl066

Wong JYY, Zhang H, Hsiung CA, Shiraishi K, Yu K, Matsuo K, Pik Wong M, Hong YC, Wang J, Seow WJ, et al. 2020. Tuberculosis infection and lung adenocarcinoma: Mendelian randomization and pathway analysis of genomewide association study data from never-smoking Asian women. Genomics 112: 1223-1232. doi:10.1016/j.ygeno 2019.07.008

Wray NR, Lin T, Austin J, McGrath JJ, Hickie IB, Murray GK, Visscher PM. 2021. From basic science to clinical application of polygenic risk scores: a primer. JAMA Psychiatry 78: 101-109. doi:10.1001/jamapsychiatry.2020 .3049

Yang J, Lee SH, Goddard ME, Visscher PM. 2011. GCTA: a tool for genome-wide complex trait analysis. Am J Hum Genet 88: 76-82. doi:10.1016/j.ajhg.2010.11.011

Yang J, Ferreira T, Morris AP, Medland SE; Genetic Investigation of Anthropometric Traits (GIANT) Consortium; DIAbetes Genetics Replication and Metaanalysis (DIAGRAM) Consortium; Madden PAF, Heath AC, Martin NG, Montgomery GW, et al. 2012. Conditional and joint multiple-SNP analysis of GWAS summary 
statistics identifies additional variants influencing com plex traits. Nat Genet 44: 369-375. doi:10.1038/ng.2213

Yengo L, Sidorenko J, Kemper KE, Zheng Z, Wood AR, Weedon MN, Frayling TM, Hirschhorn J, Yang J, Visscher PM, et al. 2018. Meta-analysis of genomewide association studies for height and body mass index in $~ 700000$ individuals of European ancestry. Hum Mol Genet 27: 3641-3649. doi:10.1093/hmg/ ddy271
Yule GU. 1902. Mendel's laws and their probable relations to intra-racial heredity. New Phytol 1: 222-238. doi:10.1111/ j.1469-8137.1902.tb07336.x

Zhou S, Butler-Laporte G, Nakanishi T, Morrison DR, Afilalo J, Afilalo M, Laurent L, Peitzner M, Kerrison N, Zhao K, et al. 2021. A Neanderthal OAS1 isoform protects individuals of European ancestry against COVID-19 susceptibility and severity. Nat Med 27: 659-667. 


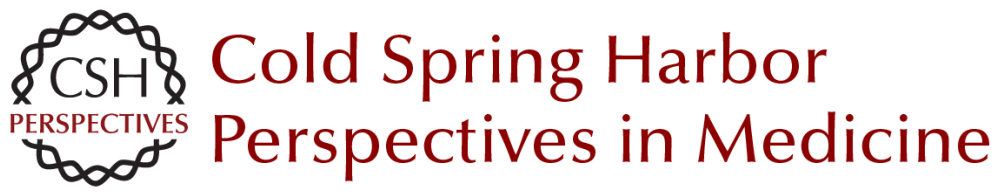

\section{Causal Inference with Genetic Data: Past, Present, and Future}

Jean-Baptiste Pingault, Rebecca Richmond and George Davey Smith

Cold Spring Harb Perspect Med published online September 27, 2021

Subject Collection Combining Human Genetics and Causal Inference to Understand Human Disease and Development

Mendelian Randomization

Ewan Birney

Human Genomics and Drug Development Amand F. Schmidt, Aroon D. Hingorani and Chris Finan

The Meaning of "Cause" in Genetics Kate E. Lynch

Using Mendelian Randomization to Improve the Design of Randomized Trials

Brian A. Ference, Michael V. Holmes and George Davey Smith

Computational Tools for Causal Inference in Genetics

Tom G. Richardson, Jie Zheng and Tom R. Gaunt

Family-Based Designs that Disentangle Inherited Factors from Pre- and Postnatal Environmental

Exposures: In Vitro Fertilization, Discordant

Sibling Pairs, Maternal versus Paternal

Comparisons, and Adoption Designs

Anita Thapar and Frances Rice

Polygenic Mendelian Randomization Frank Dudbridge
Causal Inference with Genetic Data: Past, Present, and Future Jean-Baptiste Pingault, Rebecca Richmond and George Davey Smith

Mendelian Randomization: Concepts and Scope Rebecca C. Richmond and George Davey Smith

Triangulating Evidence through the Inclusion of Genetically Informed Designs

Marcus R. Munafò, Julian P.T. Higgins and George Davey Smith

Twins and Causal Inference: Leveraging Nature's Experiment

Tom A. McAdams, Fruhling V. Rijsdijk, Helena M.S. Zavos, et al.

Integrating Family-Based and Mendelian

Randomization Designs

Liang-Dar Hwang, Neil M. Davies, Nicole M. Warrington, et al.

Causal Inference Methods to Integrate Omics and Complex Traits Eleonora Porcu, Jennifer Sjaarda, Kaido Lepik, et al.

\author{
Multivariable Mendelian Randomization and \\ Mediation
}

Eleanor Sanderson

For additional articles in this collection, see http://perspectivesinmedicine.cshlp.org/cgi/collection/ 